\title{
Training people with dementia/cognitive impairment and their carers in the use of web-based supportive technologies (Innovative practice)
}

\author{
Katie Cunnah ${ }^{1}$ David Howe ${ }^{2}$ Jonathan Thorpe ${ }^{2}$ Rosie Dunn $^{2}$ Rebecca Platt ${ }^{3}$ Caroline \\ White $^{2}$ Kevin Paulson ${ }^{4}$ Emma Wolverson ${ }^{2}$ \\ ${ }^{1}$ Department of Psychology, Faculty of Health Sciences, University of Hull, UK \\ ${ }^{2}$ Psychological Health, Wellbeing and Social Work, School of Health and Social Work, \\ Faculty of Health Sciences, University of Hull, UK \\ ${ }^{3}$ Centre for Dementia Research, Leeds Beckett University, UK \\ ${ }^{4}$ School of Engineering, Faculty of Science and Engineering, University of Hull, UK
}

\begin{abstract}
Little is known about the training and support people with dementia and their carers need to use digital solutions. This paper shares learning from a bespoke programme that successfully trained people with dementia or mild cognitive impairment and their informal carers to use a social networking website. This work was undertaken as part of the European Horizon 2020 Caregiverspro-mmd trial (ISRCTN15654731). The training methods described offer an improved understanding of how best to deliver digital-skills training that meets the needs of a diverse client group. The effectiveness of the programme is evidenced with qualitative and quantitative data.
\end{abstract}

\section{Keywords}

Dementia, cognitive impairment, carer, website, training, technology, digital

\section{Introduction}

Assistive technologies have an important role to play in meeting information needs and providing support as we embrace new and innovative solutions in dementia care (Department of Health, 2015). Healthcare technology is an active, fast-moving area of research; already in use are devices assist in activities of daily living, such as safety and security, memory enhancement, cognitive stimulation and rehabilitation (Holthe, Halvorsrud, Karterud, Hoel, \& Lund, 2018). Websites that assist in medicine management have received particular attention in recent years (e.g. Horne et al., 2018). Technological solutions have tended to focus on supporting family carers however, rather than on providing interventions aimed directly at the individual with dementia (Smith \& Mountain, 2012). This may be based on an assumption that people with dementia will struggle to use technologies.

Although the use of technology throughout the lifespan is increasing at a rapid rate, there still exists an age-related divide; many people living with dementia and those supporting them, either formally or informally, may not be existing users of technology (Rathbone \& Prescott, 2017). The barriers to daily use of available technologies include 
limited access, interest or motivation, and a lack of skills and knowledge of how to use what is on offer (Green \& Rossall, 2013). Physical and age-related factors, such as deterioration in eye-hand coordination, can also be limiting factors in the adoption of technologies. Therefore it is important that researchers and developers designing interventions in the area need to consider that access to technology and the internet may not be enough to embed digital interventions in the lives of people with dementia; appropriate and tailored support is crucial. When successfully delivered, digital skills training has been found to have a significant positive impact on people with dementia and their carers (Dementia and Digital: Using technology to improve health and wellbeing for people with dementia and their carers, 2016).

This paper shares new learning from the development of a successful training programme to support the implementation of a social networking website for people with dementia or mild cognitive impairment and their family caregivers.

\section{The Caregiverspro-mmd Website: An overview}

Caregiverspro-mmd is a Horizon 2020 pan-European study (ISRCTN15654731) investigating the efficacy of a website co-designed with people with dementia or mild cognitive impairment and their carers. The aims of the website were to provide (1) peer support (2) information about dementia and supporting people living with dementia or mild cognitive impairment, information about support services and local resources (3) pleasure / enjoyment. The website features and design were guided by feedback from focus groups and usability testing and the subsequent main features included: a newsfeed similar to that seen on social media sites such as Facebook, opportunities to interact with other users by commenting on and liking newsfeed posts, games, a journal, a calendar and a library containing information and local support and resources.

Once designed the website was tested in a randomised controlled trial across four European sites. Participants were dyads consisting of people with dementia or mild cognitive impairment and their family carers. The main outcome measures were quality of life and carer burden, with measures taken at baseline, 6 and 12 months. In addition to standardised outcome measures, website user interaction was logged including the access to individual pages and button actions such as liking posts. Each site created daily content for the website containing news stories and activities and undertook a moderating role.

\section{The UK Caregiverspro-mmd Study}

In the UK, 100 dyads were randomly assigned to the control $(n=49)$ and intervention $(n=51)$ groups. After 12 months, the drop-out rate was $25 \%$, leaving a total of 75 dyads who completed the study ( control $n=39$, intervention $n=36$ ). Mean age was 68 years in the intervention group and 67 years in the control group. Across the intervention and control groups, when withdrawals are excluded, $61.3 \%$ of people had a diagnosis of dementia and $38.7 \%$ had mild cognitive impairment. Overall, the mean Mini-Mental State Exam (MMSE) score at baseline was $22.51(\mathrm{SD}=4.63$, range 12-30). 
Upon commencing the study, data was collected about use of the internet. In the intervention group, $88.9 \%$ had an internet connection at home. At baseline, $75 \%$ of carers and $36.1 \%$ of people dementia or mild cognitive impairment said that they used the internet every day. $12.5 \%$ of carers and $13.9 \%$ people with dementia or mild cognitive impairment used it once per week, $2.8 \%$ of carers and $2.8 \%$ of people dementia or mild cognitive impairment used it once per month, and $8.3 \%$ of carers and $47.2 \%$ of people dementia or mild cognitive impairment said that they never used it.

\section{The Engagement Programme}

Given the wide variation in internet use, the UK team recognised that without sufficient engagement promotion, uptake of the Caregiverspro-mmd website was likely to be low and its potential benefits would be unlikely to be realised. The team therefore elected to include an engagement programme as a part of the intervention. The engagement programme consisted of initial one-to-one training sessions, group training, supporting documents, supportive interactions on the website, and ongoing availability of technical support. The engagement programme was delivered for the first 9 months of the 12-month study. During this time, the team trialled a range of different methods of delivery, each of which had strengths and limitations. Some were better suited at different times. The methods we found to be most useful are outlined below.

Initial one-to-one sessions. All participants in the intervention group took part in an initial 1:1 training session, to introduce them to the tablet device and the main features of the website, connect to the home broadband network if available, set them up with a profile and begin to build their network of friends. A written user manual was also provided. These sessions took approximately one hour, and two research team attended so that both the people with dementia or mild cognitive impairment and the carers had one-to-one support.

Group training. All participants were invited to attend group training sessions following the initial one-to-one training. Typically, 8-12 participants attended each session, but engagement ranged from 4 to 18 participants. Dyads were able to work with together or independently and the decision was made not to separate groups for carers and people with dementia or mild cognitive impairment. Sessions were two hours long and refreshments were provided. Participants were able to attend as many of the training groups as they wished. The sessions ran as stand-alone sessions rather than as a course, so that participants who were unable to attend at certain times did not miss out or get left behind. The research team supported attendance at training by contacting participants before sessions by telephone or via the website to remind them of dates, times and locations. Where transport was an issue, the research team offered support with getting to the training venues. The suitability and success of the training methods were continuously evaluated throughout the programme in order to modify the training delivered. A subsequent training guide was developed detailing learning and making recommendations for future digital training. The aims of the sessions were to increase confidence in using the website.

Ongoing technical support. The research team were available throughout the study to offer technical support via the website, or by email, telephone, or face-to-face. Issues that 
were raised and dealt with included difficulties with tablets not allowing users to log in, and issues with connectivity.

\section{Group Training Methods}

The engagement programme gained pace throughout the research as the research team learnt about what worked best for participants. Resources and methods were adjusted in response to participant feedback. Through this process, it became clear that some methods were particularly effective at certain points in training. A brief outline of the most effective methods of delivery are outlined below.

Microsoft PowerPoint. Initial training sessions were delivered using MS PowerPoint to guide people through the basics of using the website. These involved displaying screenshots on a projector screen and walking group members through basic actions. Following this, we asked them to repeat the task independently. For these sessions, the tables were organised as a horseshoe. Sessions lasted two hours with aims and objectives stated at the beginning, then two segments of taught input followed by independent activity with a break in between. Finally, a plenary recapped on what had been covered and reflected on challenges and successes experienced by participants. This method of delivery was successful in reminding people of the content that had been delivered in the one-to-one sessions. We also provided supportive resources in the form of 'crib sheets' - these were full colour, straightforward step-by-step guides for each major action on the website that we endeavoured to teach e.g. how to search for a friend on the website. They included screen shots with arrows illustrating each component step, large fonts and clear language. As participants started to learn how to use the website, participants were moving at different rates and a new method of delivery was needed to prevent some feeling left behind and others disengaging through inadequate challenge. We therefore developed the Task Card method.

Task card method. The task card method involved following a series of laminated cards, each presenting an activity with a series of three differentiated difficulty levels which were simply termed and colour coded 'easy' (green), 'medium' (amber) and 'hard' (red). We created six task cards, and each had supporting crib sheets with step-by-step guides for completing the tasks. This method facilitated independence by scaffolding learning. It enabled learners to self-differentiate by working at their own pace and selecting supportive resources to help them complete tasks if they needed them, or to work without them if they did not. They were also able to take these resources home so that they could repeat the activities at their own convenience. This was also particularly conducive to fostering independence and retention of learning, as well as increasing participants' confidence in using the tablets on their own.

Extending learning. A further 16 'challenge cards' were developed. The activities on the challenge cards required participants to repeat procedures they had already practiced using the task cards, but extended their learning by encouraging them access more complex features of the website, as well as encouraging engagement with external resources such as information websites and video resources. In the final months of the training programme we 
also created 'advanced challenges' which were a series of five activities requiring greater autonomy and independence in accessing and using a range of resources via the website.

Stamp booklets. All of the task cards, challenge cards, and advanced challenges were accompanied by stamp booklets in which each learner could stamp a task as they completed it. This enabled participants to keep track of their progress and was also a useful motivational strategy, as well as helping them to recap at each session on what they had already done and what they still needed to do. Participants were however free to repeat tasks whenever they wanted or needed to. We found that participants really enjoyed keeping track of their progress, and gained a sense of achievement from completing the stamp booklets. It helped them to remember from one session to the next what they could already do, and they often compared their progress with each other which kept them motivated.

Room layout \& session structure. For sessions delivered via the task card method, participants were seated on tables of four. The sessions began with a brief introduction, included a refreshment break half way through, and as a plenary we organised group activities such as 'group chats' and quizzes. These always required interaction via the website. For example, the group chat involved a question being posted on the newsfeed by the research team, such as 'If you were a biscuit, what would you be and why?', and all participants replied by commenting on the post. This modelled posting, commenting and posing questions on the newsfeed, but also encouraged interaction and fun within the group when the more general delivery method had become more individualised and required less social interaction.

\section{How the training supported engagement with the website}

The uptake of group training was incredibly high; $71 \%$ of dyads attended at least one group training session, and $63.2 \%$ attended two or more training sessions. The number of group training sessions attended was associated with an increase in the number of visits to the website. The mean number of visits to the website in a six month period for individuals who attended 0-1 group training session was 9.00 (SD 11.84). When compared with user data for individuals who attended $2+$ group training sessions $(n=158.60, S D 157.20)$, there were significantly more visits in the latter group $(U=126, p<.001)$.

Attendance at group training sessions was associated with increased activity on the website. This data was recorded as 'actions'. An 'action' is any activity on the website whereby there is a change, for example, changing page, commenting, liking, favouriting, adding a friend, posting on the newsfeed, creating an appointment, and opening a game. The mean number of actions performed on the website for individuals who attended 0-1 group training session was 101.29 ( $S D$ 99.65). When compared with user data for individuals who attended $2+$ group training sessions $(n=1510.44, S D$ 1573.83), there were significantly more actions performed on the website by the $2+$ group than the $0-1$ group $(U=132, p<.001)$.

Those who attended two or more training sessions spent more time on the website overall. The mean amount of time in seconds spent on the website by the individuals who attended 0-1 group training session was 5228.07 (SD 5188.63). When compared with user data for individuals who attended $2+$ group training sessions ( $n=83457.15, S D$ 85196.48), the $2+$ group spent significantly more time on the website than the 1-0 group $(U=154, p<.001)$. 


\section{Participant perspectives}

Qualitative data suggests that training sessions provided important functions beyond increasing familiarity with the technology. Those who attended training enjoyed attending a group where there was a clear sense of purpose, they were learning something, and they gained a sense of achievement, but there was also the social element that made them feel supported. Because of this sense of purpose, the training also attracted some people who did not usually enjoy going to groups:

“... 'Cause there's a reason for coming, isn't there, and the reason is as you... find out how to use this and get more information out of it and use it in a different way plus you get your, your friendship with your, your other people but we don't get to talk much, do we? 'Cause you're on all this... 'cause you keep us directed on the learning bit." (Carer)

"You do need the group training. Yeah, you do because people forget. You know, they come back this month and they've forgot [sic] what you told them last month." (Person with dementia/mild cognitive impairment)

Both carers and people with dementia or mild cognitive impairment highlighted that repetition in learning the new technology was crucial for them, and being able to do so at their own pace, as many times as they required, for example:

"I told him off because he were [sic] rushing ahead and I hadn't got the hang of it... and he was saying 'but you've done it before,' I'd say 'I don't care, I'm doing it again." "(Carer)

"Persistence, persistence... after finding it very...making mistakes and getting frustrated at first, I still do at times, but gradually I've got more into it and found it more interesting." (Person with dementia/mild cognitive impairment)

Participants found the written resources that supported learning particularly helpful. They valued the fact that these were revised according to the needs of the groups:

"When I first started doing it, I asked about these, and then they produced these crib sheets and they were brilliant. That was the thing that, if you like, made it a lot easier... because, knowing if I get a problem, I can dig my file out and go through it again." (Carer)

"I've got all the, you know, the crib sheet, the papers that you've given us, and if I want to maybe try and take a photo, I have to go back to that. I don't remember kind of how to do it." (Person with dementia/mild cognitive impairment) 
"You revised those, didn't you, from the very, very first ones that you... of the handouts that you did, you revised them maybe more, sort of user friendly? "(Carer)

The provision of training and resources that enabled gently building on knowledge was of value to the participants in the development of digital autonomy and confidence:

“...When you first got it you only had a set amount of information so you just went on what you knew but gradually coming and building up every week we can actually manage it now and we can do more or less what we want, can't we?" (Carer)

"Because you get told here how to use it and then you go home, you've forgotten it by then but we, you had the handouts what [sic] went with it, that we logged in and things, I found it great from there." (Person with dementia/mild cognitive impairment)

However, not all participants engaged in the group training sessions regularly. Although efforts were made to ensure sessions were as accessible and supportive as possible, accessibility remained one of the main barriers to attendance, as did difficulties with physical health:

"...Although that is a bit of a way to go, and erm, if (carer) wanted the car, then I can't really get there, not from here... I mean as I say I can drive but of course, (carer) wants it sometimes and you can't just get a bus, well you can get a bus more to town here but not anywhere else". " (Person with dementia/mild cognitive impairment)

"I've had, like, nearly three weeks in hospital out of it, so I'm a bit behind" (Person with dementia/mild cognitive impairment)

Not all participants liked the written resources:

“[The stamp booklets are] childish. Yeah, it's like being at school." (Person with dementia/mild cognitive impairment)

Some participants commented that the website and the training would have been more useful to them at earlier stages of their journey through dementia:

"I think I would have used it more if it had been five, six years ago...I think I'd have put a lot more on 'cause I, at that stage, with anything that happened, [I thought] 'oh, can anybody help me?', (Carer) 
However, of those who did attend group training, a number commented on how much more confident they felt using other types of technology having engaged with the Caregiversprommd training programme, and generally wanted to continue to use digital resources on an ongoing basis:

"[It was] Confusing to begin with. Erm... 'cause it was all new and... but I, I mean we had iPads anyway but I found it so different to using an iPad...I had an iPad that I couldn't use and I couldn't master it... [I] didn't touch the iPad until we came to the first training session. Well, my iPad was at home and my grandchildren used to play on it. They'd say 'Oh come on, Grandma, I'll show you' but I never learnt because it's...well I can't keep up with that...I can now... Because you talked me through it where they just showed me and I couldn't pick up just being shown." (Carer)

The benefits of developing digital skills and confidence have the potential to reduce feelings of isolation and increase feelings of connectedness:

"I've got closed in since I retired... and that [the platform] took me out of my own world and into other people's. And not just other people's but what's going on in the world as much as anything...You don't realise how much you get isolated...I used to run a cricket club... and gradually I shrunk away from that. That's not necessarily due to the dementia, that's my personality...[the platform] has put a new perspective on things and what I should be doing now, whether I do is a different matter but it certainly told me what I should be doing (laughter)." (Person with dementia/mild cognitive impairment)

\section{Discussion}

Because participants preferred to meet people face-to-face, those who attended the most training sessions tended to have a wider network and increased activity on the website. The findings suggest that attendance at multiple training sessions was associated with increased user engagement. Although causality cannot be inferred from this result, the positive correlation between number of training sessions attended and number of visits, actions and time spent on the website suggests that the delivery of a group training programme specially designed for people with dementia or mild cognitive impairment and their carers may successfully engage this group in new technologies. Without the element of group training, engagement is unlikely to be as successful and the potential benefits of habitual use of the technology less likely to be realised.

However, the limitations of group training must also be considered. Those who are willing and able to attend and who are comfortable learning in a group environment may not be those who most in need of social interaction or supportive technologies. For those who are unable or prefer not to attend group training, they may be more likely to disengage from such programmes completely. This suggests that attendance at group training is a key component in increasing user engagement generally in technology implementation programmes for people with dementia or mild cognitive impairment and their carers, but that other methods of 
engagement must be explored in order to reach the most socially isolated, so that they may reap the same benefits from using digital technologies as those who are more active socially. Despite this, the evidence suggests that providing a website alone is not enough to meet the needs of this group - training that offers opportunities for repetition, varied challenge, offline interaction and is tailored to range of experience and abilities should also be offered.

\section{Conclusion}

Health and social care increasingly recognises the need for investment in digital technologies to support the delivery of care. Innovative studies have investigated the use of a range of digital interventions to support care management and to increase social support for people with dementia or mild cognitive impairment and their carers. However, our research suggests that maximum benefits are realised when the process of engagement is carefully planned. Attendance on a well-planned training course increases user confidence and makes it more likely that those individuals will continue working with technology. As the selection of digital interventions available in dementia care broadens, there is a need for commissioners and service providers to recognise the value of high-quality training and engagement packages, and to ensure that these are embedded and integral, if the benefits of the technologies are to be fully realised.

\section{Recommendations}

We have published a Training Guide that provides evidence-based recommendations for the delivery of training in digital technologies for people with dementia or mild cognitive impairment and their carers. It is available on the Yorkshire and Humber Clinical Network site http://www.yhscn.nhs.uk/mental-health-clinic/Dementia/dementia-documents-andlinks.php\#D

\section{Conflict of interest statement}

None declared.

\section{References}

Dementia and Digital: Using technology to improve health and wellbeing for people with dementia and their carers. (2016). Retrieved from https://www.goodthingsfoundation.org/sites/default/files/researchpublications/dementia_and_digital.pdf

Department of Health. (2015). Prime Minister's challenge on dementia 2020. Retrieved from https://assets.publishing.service.gov.uk/government/uploads/system/uploads/attachment data/file/414344/pm-dementia2020.pdf

Green, M., \& Rossall, P. (2013). Digital inclusion evidence review. Retrieved from https://www.ageuk.org.uk/globalassets/age-uk/documents/reports-andpublications/reports-and-briefings/activecommunities/rb_sept13_age_uk_digital_inclusion_evidence_review.pdf 
Holthe, T., Halvorsrud, L., Karterud, D., Hoel, K.-A., \& Lund, A. (2018). Usability and acceptability of technology for community-dwelling older adults with mild cognitive impairment and dementia: a systematic literature review. Clinical Interventions in Aging, Volume 13, 863-886. https://doi.org/10.2147/CIA.S154717

Horne, F., Burns, P., Traynor, V., Gillespie, R., Mullan, J., Baker, A., ... Win, K. T. (2018). Managing medications for individuals living with a dementia: Evaluating a web-based information resource for informal carers. International Journal of Older People Nursing, 13(3), e12198. https://doi.org/10.1111/opn.12198

Miranda-Castillo, C., Woods, B., \& Orrell, M. (2013). The needs of people with dementia living at home from user, caregiver and professional perspectives: a cross-sectional survey. BMC Health Services Research, 13(1), 43. https://doi.org/10.1186/1472-6963$13-43$

O’Connor, A., Crowley, F., Vaughan, D., \& O’Sullivan, S. S. (2017). PO036 The readability of internet information on lewy body dementia. Journal of Neurology, Neurosurgery \& Psychiatry, 88(Suppl 1), A21.3-A21. https://doi.org/10.1136/jnnp-2017-ABN.71

Rathbone, A. L., \& Prescott, J. (2017). The use of mobile apps and SMS messaging as physical and mental health interventions: systematic review. Journal of Medical Internet Research, 19(8), e295. https://doi.org/10.2196/jmir.7740

Smith, S. K., \& Mountain, G. A. (2012). New forms of information and communication technology (ICT) and the potential to facilitate social and leisure activity for people living with dementia. International Journal of Computers in Healthcare, 1(4), 332. https://doi.org/10.1504/IJCIH.2012.051810

van der Roest, H. G., Meiland, F. J. M., Comijs, H. C., Derksen, E., Jansen, A. P. D., van Hout, H. P. J., ... Dröes, R.-M. (2009). What do community-dwelling people with dementia need? A survey of those who are known to care and welfare services. International Psychogeriatrics, 21(05), 949. https://doi.org/10.1017/S1041610209990147 